\title{
First Principles Study of Cerium Monochalcogenides
}

\author{
Recep Eryiğit \\ Faculty of Engineering, Ankara University, 06100 Ankara, Turkey \\ Correspondence should be addressed to Recep Eryiğit; reryigit@eng.ankara.edu.tr
}

Received 18 March 2014; Accepted 6 May 2014; Published 21 July 2014

Academic Editor: Dario Alfe

Copyright (C) 2014 Recep Eryiğit. This is an open access article distributed under the Creative Commons Attribution License, which permits unrestricted use, distribution, and reproduction in any medium, provided the original work is properly cited.

\begin{abstract}
A theoretical investigation of structural, magnetic, electronic, and lattice dynamical properties of cerium monochalcogenides using the generalized gradient approximation $(\mathrm{GGA})+U$ within ultrasoft pseudopotentials and a plane-wave basis is presented. All the calculated quantities, except the local magnetic moments, are found to be in good agreement with the experimental data. The lattice dynamical results indicate a strong chalcogenide dependence for the anomalous features of the phonon dispersions.
\end{abstract}

\section{Introduction}

The single $4 \mathrm{f}$ electron of cerium element gives rise to many anomalous and fascinating properties in cerium compounds, such as heavy-fermion systems, intermediate valence compounds, Kondo metals, and Kondo insulators. Although the energy of this $4 \mathrm{f}$ electron is in the range of $5 \mathrm{~d}$ and $6 \mathrm{~s}$ valence electrons, its wave function is spatially localized tighter than semicore $5 \mathrm{~s}$ and $5 \mathrm{p}$ electrons. The competition between cerium $4 \mathrm{f}$ electron being itinerant or localized determines the character of many cerium compounds. Cerium monochalcogenides $(\mathrm{CeX}, \mathrm{X}=\mathrm{S}, \mathrm{Se}, \mathrm{Te})$ are some of the structurally simplest materials which show interesting electronic and magnetic properties due to the existence of a $4 \mathrm{f}$ electron of cerium. CeX compounds crystallize in $\mathrm{NaCl}$ structure at ambient conditions and exhibit a type II antiferromagnetic order below $T_{M}=8.7,5.4$, and $2.2 \mathrm{~K}$ for CeS, CeSe, and $\mathrm{CeTe}$, respectively. The lowest-lying $J$ multiplet ${ }^{2} \mathrm{~F}_{5 / 2}$ of $\mathrm{Ce}^{3+}$ ion is split into a ground state doublet $\Gamma_{7}$ and an exited state quartet $\Gamma_{8}$ by the cubic crystalline electric field. The saturation magnetic moments are considerably reduced below a value of $0.71 \mu_{B}$ which is expected from ground $\Gamma_{7}$ state [1]. All three compounds undergo structural phase transitions from $\mathrm{NaCl}$ to $\mathrm{CsCl}$ structure under pressure. Phonon dispersion of $\mathrm{CeX}$ also exhibits strong anomalies in the longitudinalacoustic branch, especially near the L point, where it crosses the transverse ones for CeSe [2].

Electronic and lattice dynamical properties of cerium monochalcogenides have been studied theoretically and experimentally by several groups over the years [1-10]. De and Chatterjee [3] used a self-consistent linear augmented plane-wave method in the local-density approximation to study the change in valency with pressure for CeS. Svane et al. [4] investigated the electronic structure of rare-earth chalcogenides and pnictides with a self-interaction-corrected local-spin-density approximation within linear muffin tin orbital method along the lines of [3]. Price et al. carried out an LSDA and LDA $+U$ study of magnetooptic properties of $\mathrm{CeSb}$ and $\mathrm{CeTe}$ in full potential linear muffin-tin orbital scheme and found that the LDA $+U$ calculation fails to capture the essential elements of the electronic structure of $\mathrm{CeTe}$, since it failed to obtain a correct magnetic moment [5]. Collins et al. [9] investigated the peculiar magnetic properties of cerium pnictides and chalcogenides by using a model Hamiltonian with LDA derived parameters and LSDA approaches and found that the model was able to reproduce the experimentally observed moment collapse while LSDA and LDA $+U$ were not able to predict even the ordering of the magnetic moments of $\mathrm{CeS}, \mathrm{CeSe}$, and CeTe. The lattice dynamics of CeSe were investigated by Steiner et al. by using the nonorthogonal tight-binding method of Varma and Weber to explain the longitudinal acoustic phonon softening [2]. Antonov et al. presented a review of experimental and theoretical works on optical and polar Kerr spectra of CeX compounds [10].

To the best of our knowledge, there is no extensive first principles study of electronic and lattice dynamical properties of cerium monochalcogenides. In the present work, we have 


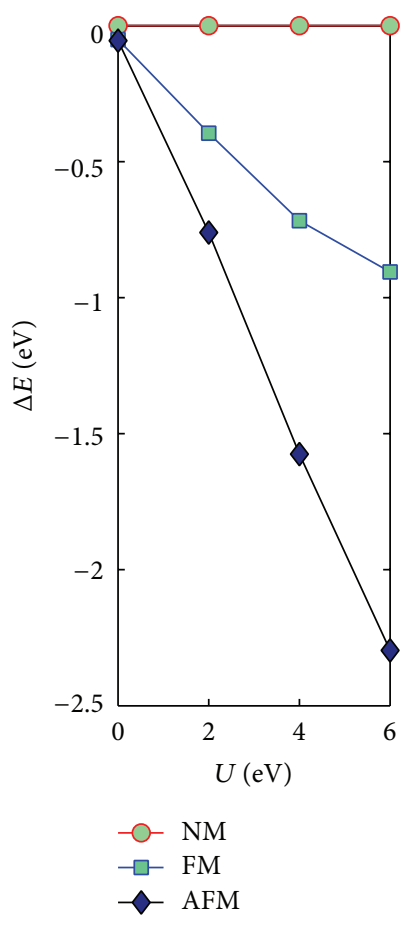

(a)

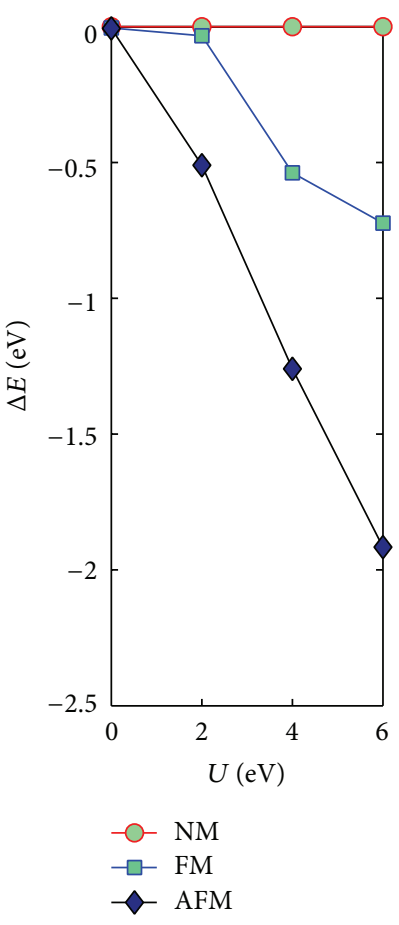

(b)

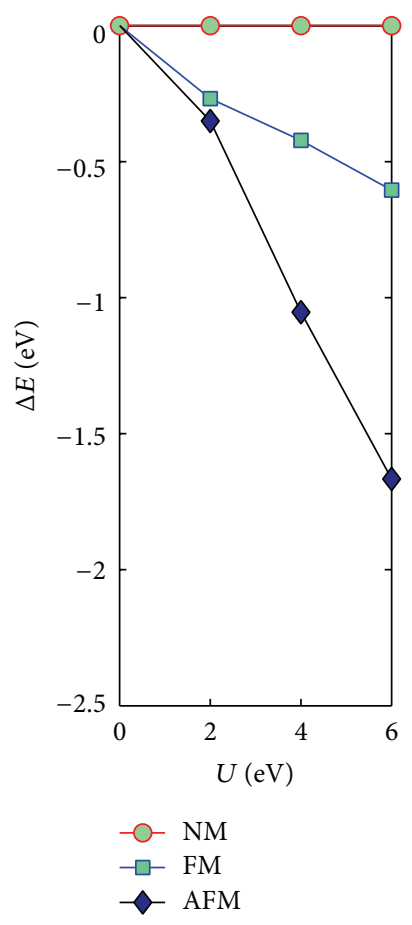

(c)

FIgURE 1: The difference in energy of the different magnetic configurations as function of $U$ for (a) CeS, (b) CeSe, and (c) CeTe.

TABLE 1: Calculated lattice parameter $a_{0}$ (in $\AA$ ), bulk modulus $B_{0}$ (in GPa), pressure derivative of the bulk modulus $B^{\prime}$, and magnetic moment (in $\mu_{B} / \mathrm{Ce}$ ) for $\mathrm{CeS}, \mathrm{CeSe}$, and CeTe.

\begin{tabular}{|c|c|c|c|c|}
\hline Material & $a(\AA)$ & $B_{0}(\mathrm{GPa})$ & $B^{\prime}$ & $\mu\left(\mu_{B} / \mathrm{Ce}\right)$ \\
\hline \multicolumn{5}{|l|}{$\mathrm{CeS}$} \\
\hline Theory (this work, NM) & 5.68 & 88.4 & 4.65 & \\
\hline Theory (this work, FM) & 5.68 & 85.4 & 5.16 & $0.01,1.15[16]$ \\
\hline Theory (this work, AFM) & 5.69 & 85.0 & 5.29 & $0.01,1.42[16]$ \\
\hline Theory [17] & 5.73 & 87.0 & 4.83 & \\
\hline Theory [13] & 5.78 & 96.0 & 4.70 & \\
\hline Exp. [18] & 5.77 & 82.0 & 2.2 & $0.31[10]$ \\
\hline \multicolumn{5}{|l|}{ CeSe } \\
\hline Theory (this work, NM) & 5.91 & 75.1 & 4.62 & \\
\hline Theory (this work, FM) & 5.92 & 66.9 & 4.89 & $0.51,1.12[16]$ \\
\hline Theory (this work, AFM) & 5.92 & 67.0 & 4.98 & $0.52,1.47[16]$ \\
\hline Theory [17] & 5.96 & 74.4 & 4.77 & \\
\hline Theory [13] & 5.98 & 83.4 & 5.20 & \\
\hline Exp. [18] & 5.99 & 76.0 & 5.00 & $0.45[10]$ \\
\hline \multicolumn{5}{|l|}{$\mathrm{CeTe}$} \\
\hline Theory (this work, NM) & 6.32 & 57.3 & 4.62 & \\
\hline Theory (this work, FM) & 6.35 & 51.4 & 4.44 & $0.98,1.28[16]$ \\
\hline Theory (this work, AFM) & 6.35 & 51.0 & 4.52 & $0.97,1.36[16]$ \\
\hline Theory [17] & 6.36 & 58.2 & 5.15 & \\
\hline Theory [13] & 6.34 & 68.3 & 4.80 & $1.38[5], 0.08$ [19] \\
\hline Exp. [20] & 6.36 & 52.8 & 13.6 & $0.66[10]$ \\
\hline
\end{tabular}



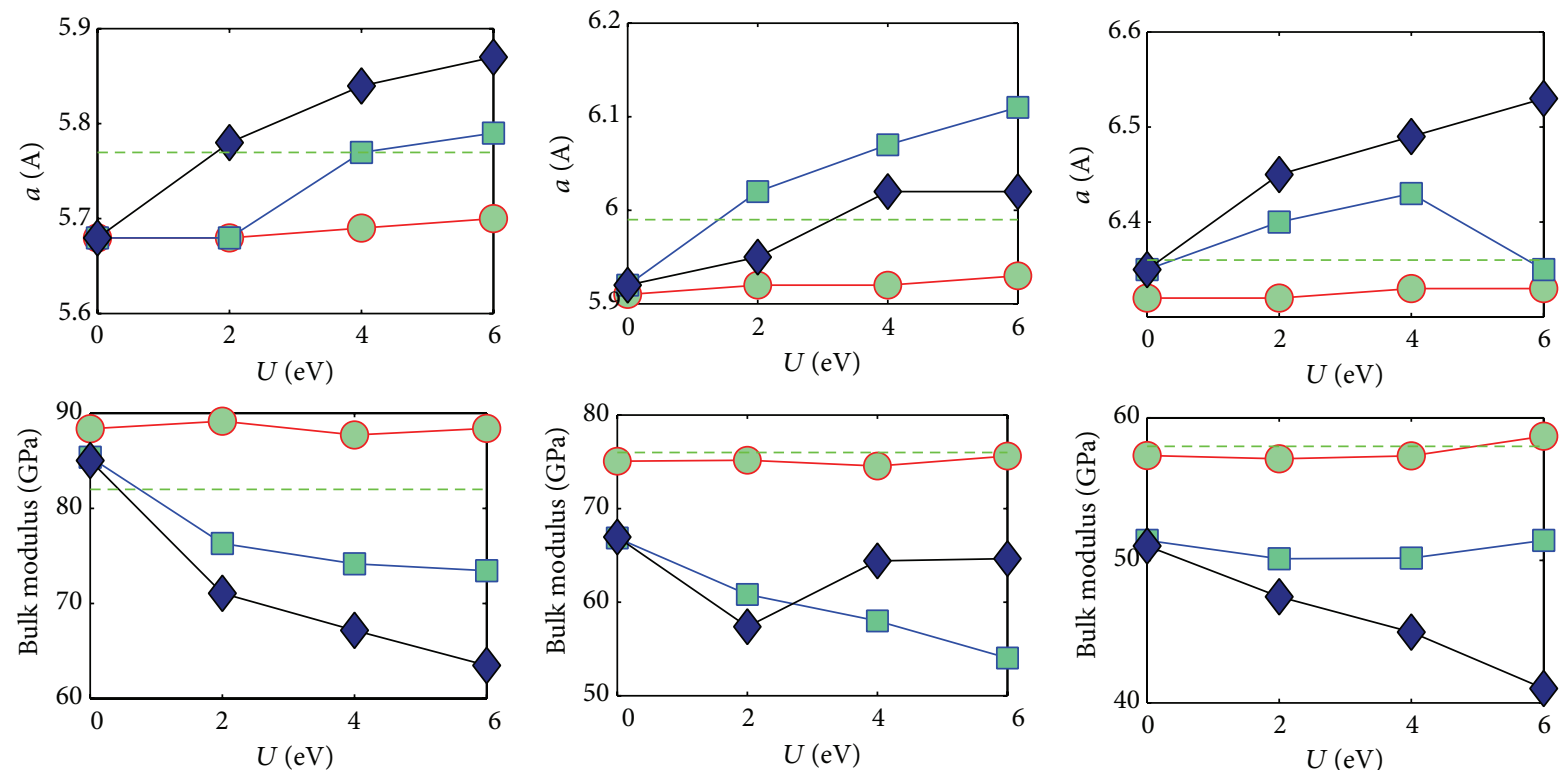

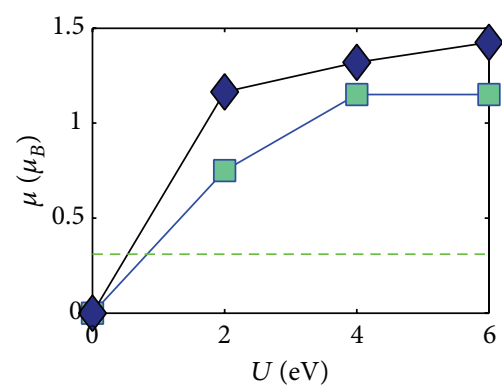

(a)

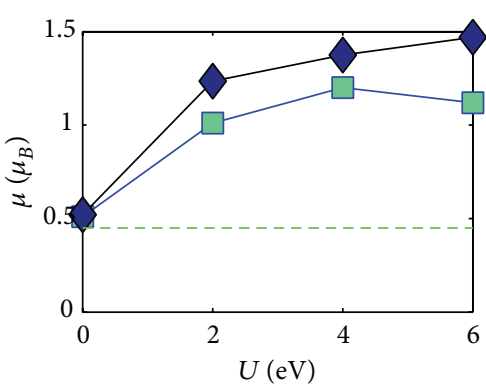

(b)

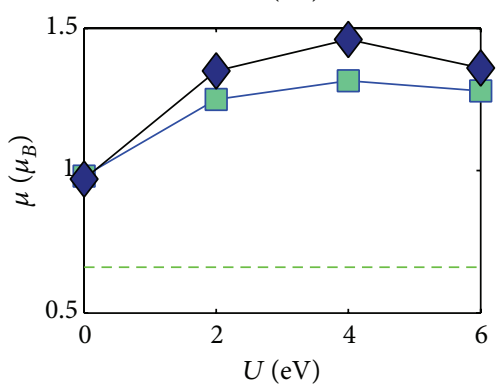

(c)

FIgURE 2: $U$ dependence of the equilibrium lattice constant (first row), bulk modulus (second row), and magnetic moments (third row) for (a) CeS, (b) CeSe, and (c) CeTe. Circles (nonmagnetic), squares (ferromagnetic), and diamonds (antiferromagnetic) are obtained from a fit of the static energy at different lattice constants to a third order Vinet equation of state for the lattice constant and the bulk modulus and the calculated equilibrium magnetic moments for the ferro- and antiferromagnetic configurations (third row). In all subplots, the dotted thick line represents the experimental value of the plotted quantity which is quoted in Table 1.

carried out extensive first principles calculations on lattice, electronical, magnetic, and lattice dynamical properties of cerium monochalcogenides in the framework of density functional and density functional perturbation theory at spin polarized GGA and GGA $+U$ levels. The outline of the paper is as follows. The details of the computational method is given in Section 2. The calculated lattice constant, bulk modulus, magnetic moment, electronic band structure, Fermi surface, and phonon dispersion relations are discussed in Section 3. The paper concludes with a brief discussion of the main findings in Section 4.

\section{Structure and the Computational Details}

All the three chalcogenides, namely, CeS, CeSe, and CeTe, considered in the present work crystallize in the $\mathrm{NaCl}$ type structure (B1) with space-group symmetry Fm3 m under normal conditions. The cerium atom is positioned at $(0,0,0)$ and the chalcogenide atom at $(1 / 2,1 / 2,1 / 2)$ reduced coordinates. The antiferromagnetically ordered phase corresponds to a stacking of ferromagnetic (111) planes with the magnetic moments lying along the [111] direction [6]. This AFM type II ordering leads to primitive unit cell with rhombohedral symmetry (space group R-3 m, group number 166) [7]. The primitive rhombohedral unit cell contains two Ce located at $(0,0,0)$ and $(1 / 2,1 / 2,1 / 2)$ and two $\mathrm{X}$ atoms placed at $(1 / 4,1 / 4$, $1 / 4)$ and $(3 / 4,3 / 4,3 / 4)$ reduced coordinates.

All the quantities reported here were obtained with the Quantum Espresso suit [11] of programs which implement density functional and density functional perturbation theory. The generalized gradient approximation to the exchange correlation functional of Perdew et al. [12] is used. We used the pseudopotentials Cefld1-pbe-rcl.0.uspp. UPF, S.pbe-vanak. UPF, Se.pbe-van. UPF, and Te.pbe-rrkj. UPF from http://www.quantum-espresso.org/. A planewave cutoff of $60 \mathrm{Ry}$ and a Monkhorst-Pack grid of $8 \times 8 \times 8$ for the Brillouine zone integrations are found to converge all the reported quantities. For the linear response phonon calculations, we use a $8 \times 8 \times 8 q$-grid to calculate the force constants which are Fourier transformed to obtain 
the interatomic force constant that are used to obtain the phonon dispersions along high symmetry directions of the first Brillouine zone.

\section{Results}

3.1. Lattice Structure and Magnetic Properties. First, we consider the equilibrium lattice structure and magnetic moment of CeX compounds and calculate the total static energy for nonmagnetic, ferro- and antiferromagnetic configurations at 12 different volumes located symmetrically around the experimental volume of the compound. Although non- and ferromagnetic phases can be described by a $\mathrm{NaCl}$ type primitive unit cell, we do all calculations in antiferromagnetic rhombohedral cell to avoid possible sampling errors in comparison of total energies. The computed volume-energy pairs are fitted to a third order Vinet equation of state to determine equilibrium lattice constant, bulk modulus, and pressure derivative of the bulk modulus of each compound and the results are displayed in Table 1 along with calculated magnetic moments and available experimental data as well as some of the findings of previous calculations. The agreement between our calculated lattice constant and the experimental values and the previous calculations is satisfactory; $a$ of $\mathrm{CeS}$ and CeSe are underestimated $\approx 1 \%$ for all magnetic configurations while $a$ of nonmagnetic CeTe are underestimated by $0.75 \%$. Lattice constants for ferro- as well as antiferromagnetic structure for all three compounds are found to be slightly higher than those calculated for the nonmagnetic phase. The effect of spin polarization on the bulk modulus is found to be more pronounced; it leads to a softening of the material for all three compounds. While this softening makes the computed bulk modulus closer to the experimental $B_{0}$ for CeS, bulk modulus of spin polarized CeSe and CeTe is found to be $\approx 10 \%$ lower than that of nonmagnetic phase which are already very close to the corresponding experimental values. Available experimental data for the pressure derivative of bulk modulus for CeS (2.2) and CeTe (13.6) are, unusually, low and high, respectively. Our calculated values for these two quantities are in the range 4.44-5.22 which is similar to other theoretical studies and more inline with general $B^{\prime}$ values.

All three cerium chalcogenides considered in the present study are known to have antiferromagnetic type II order below $T_{M}=8.7,5.4$, and $2.2 \mathrm{~K}$ with saturation sublattice moments of $0.31,0.45$, and $0.66 \mu_{B} / \mathrm{Ce}$ for CeS, CeSe, and CeTe, respectively [10]. Our calculated magnetic moments for all three compounds, especially for $\mathrm{CeS}$ and $\mathrm{CeTe}$, are unsatisfactory. The anomalous magnetic moment reduction and failure of DFT to describe it well might be related to the role of Kondo screening which cannot be accounted for in DFT $[13,14]$ or the f-band hybridization, f-band Coulomb exchange, and crystal field interactions which can be better explained with Hamiltonian systems with ab initio determined parameters [9]. To further elucidate the magnetic structure, we include strong correlation effects by repeating the calculations mentioned above in the GGA $+U$ framework for $U=2,4,6 \mathrm{eV}$ and display the resulting relative energy and structural and magnetic parameters as function of $U$ in

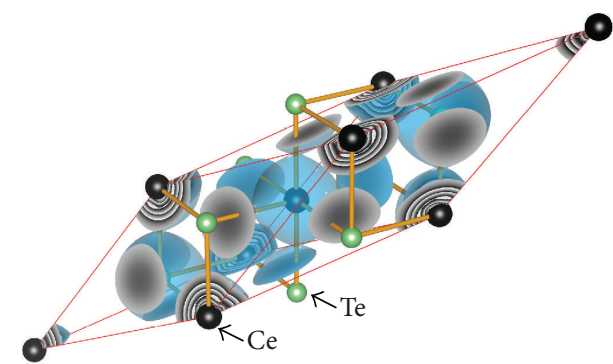

(a)

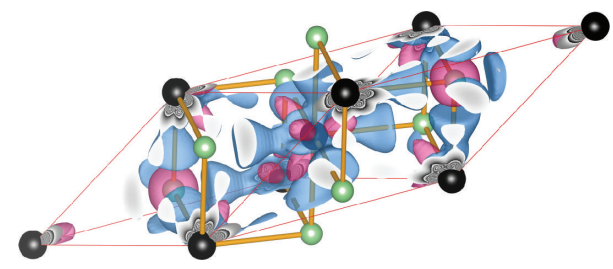

(b)

FIgure 3: Total (a) and bonding charge (b) density of CeTe in the spin-polarized rhombohedral structure. In (a) the blue surface indicates 0.005 electron $/ \mathrm{au}^{3}$ level of density and grey regions indicate intersection between the constant density surface with the primitive unit cell surface. Blue (red) surface indicates +0.003 $(-0.003)$ electron $/ a u^{3}$ density difference between CeTe and sum of separate $\mathrm{Ce}$ and $\mathrm{Te}$ atoms in the same structure in (b).

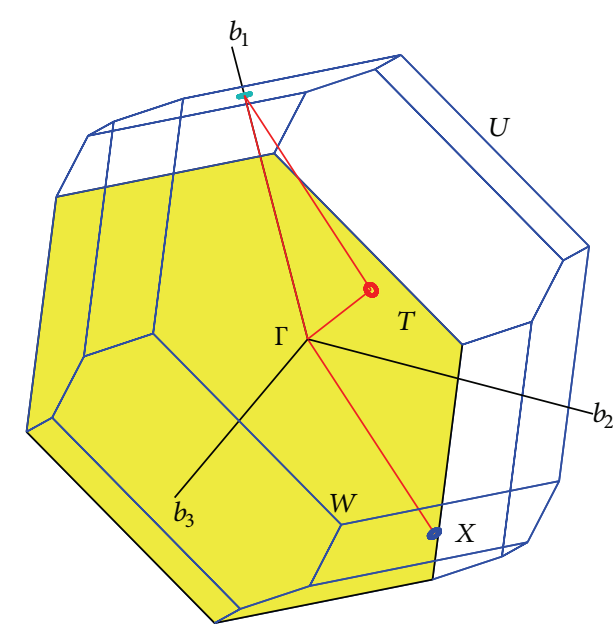

FIGURE 4: First Brillouine zone of the rhombohedral structure (the path of electronic band structure is indicated with the red line).

Figures 1 and 2, respectively. In Figure 1, we plot $U-\Delta E=E-$ $E_{\text {min }}$, where $E_{\text {min }}$ is the minimum of the total energy among all three considered magnetic structures and is calculated at the equilibrium lattice constant for the given $U$ value. From Figure 1, it is obvious that the antiferromagnetic structure has the lowest energy configuration for all considered nonzero $U$ corrections. At $U=0$, the energy difference between the various phases is not resolvable from the plot; we have found that $\mathrm{AFM}<\mathrm{FM}<\mathrm{NM}$ order is also valid at $U=0$ with $\Delta E \approx 8 \mathrm{meV}$ for all three compounds. One should note that this difference is on the order of the systematic errors of DFT. 


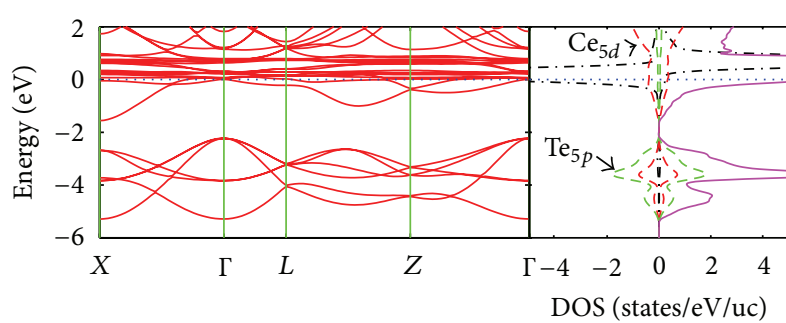

(a)

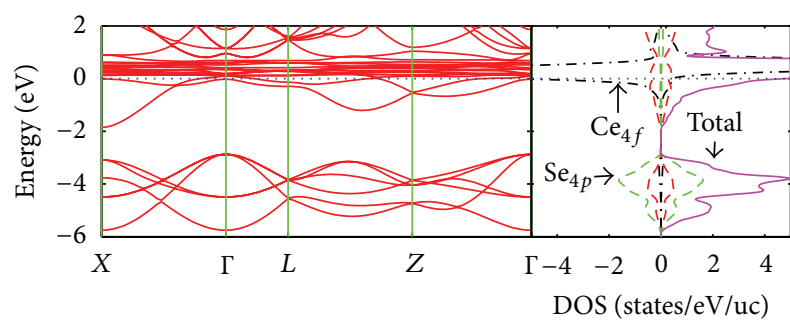

(b)

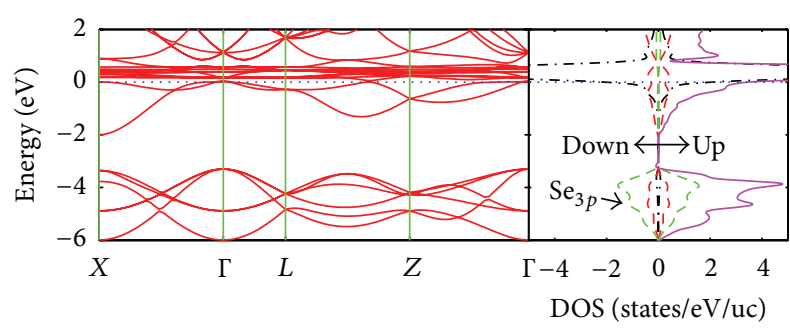

(c)

Figure 5: Electronic band structure along high symmetry lines of the first Brillouine zone and total and the partial densities of electronic states for (a) CeS, (b) CeSe, and (c) CeTe.

The $U$ dependence of lattice parameters and magnetic moments is displayed in Figures 2(a)-2(c) for CeS, CeSe, and $\mathrm{CeTe}$, respectively. The overall effect including Hubbard $U$ correction is found to be an underbinding which is reflected in increasing lattice constant and decreasing bulk modulus with increasing $U$. The reason for the effect is related to the fact that the Hubbard term leads to more localization of the 4 f electrons which no longer participate in bonding [15]. Since the lattice parameters are already well reproduced at $U=$ 0 , inclusion of the Hubbard term leads to a worsening of the agreement between the experimental and the calculated quantities as can be seen from Figure 2, especially for the bulk modulus (middle row of the figure). In an overall consideration, nonmagnetic configuration reproduces the experimental lattice constant and bulk modulus values better than ferro- and antiferromagnetic structures.

In Figure 3(a), we display the calculated total electronic charge density of CeTe in the rhombohedral structure as an example of the rearrangement of the electrons in forming cerium monochalcogenides. Figure 3(a) is the threedimensional contour plot of electronic charge density at 0.005 electron $/ a u^{3}$ level. One can discern almost spherical charge density around cerium atom in the center of the figure. Each cerium is bonded to six nearest neighbor tellurium atoms

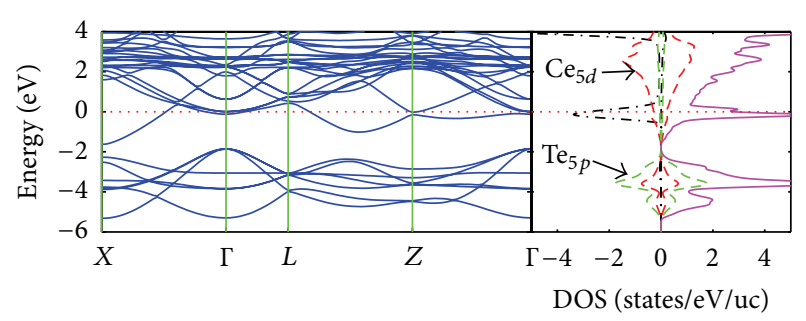

(a)

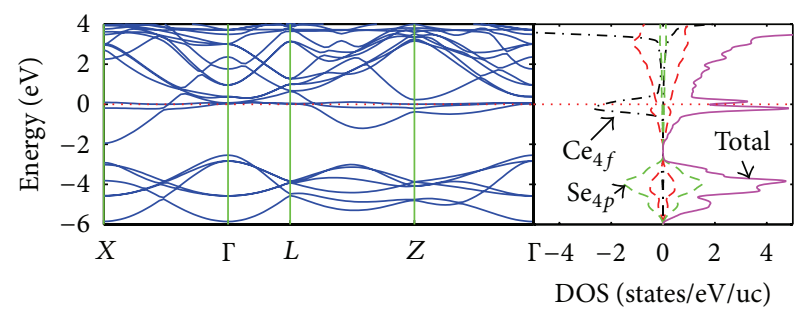

(b)

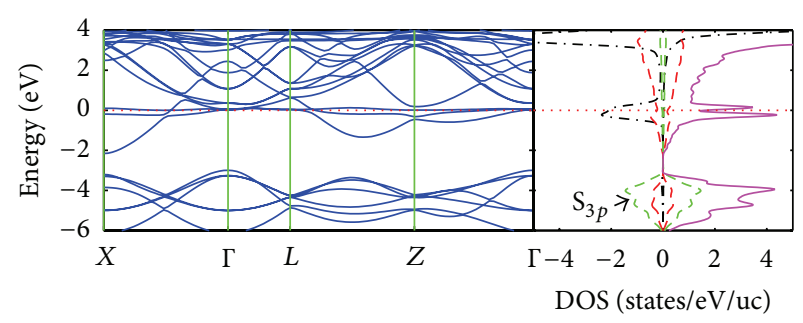

(c)

Figure 6: Same as 5 for GGA $+U$ with $U=6 \mathrm{eV}$.

which also have somewhat smaller and near spherical charge density around them. The bonding charge density defined as the difference between the converged charge density of the crystalline structure and the sum of separate atomic charge densities can give an indication of the bonding due to the crystal potential. We display the calculated bonding charge density of CeTe in Figure 3(b) as a three-dimensional contour plot at \pm 0.003 electrons $/ \mathrm{au}^{3}$ levels. The red surface indicates a decrease in the density in forming the crystal and is found around $\mathrm{Ce}$ (eight nodal ellipsoids) and $\mathrm{Te}$ (almost spherical) atoms in the figure. The blue surface shows the positive bonding charge density which is formed by the charge depleted from the red area. It can be observed from Figure 3 that electrons from $4 \mathrm{f}$-orbitals of $\mathrm{Ce}$ and $\mathrm{p}$ orbitals of Te undergo substantial geometrical change to form directional bonds between cerium and tellurium.

3.2. Electronic Band Structure. The first Brillouine zone of the rhombohedral structure used in the present calculations is shown in Figure 4. The electronic band structure of CeS, $\mathrm{CeSe}$, and $\mathrm{CeTe}$ in the antiferromagnetic phase along the $\mathrm{X}-\mathrm{\Gamma}-\mathrm{L}-\mathrm{T}-\Gamma$ directions of the first Brillouine zone of the rhombohedral lattice is shown along with the total and partial densities of electronic states in Figures 5(a)-5(c). The overall structure for all three compounds is very similar; bands formed by chalcogenide $\mathrm{p}$ hybridization with cerium $5 \mathrm{~d}$ states 

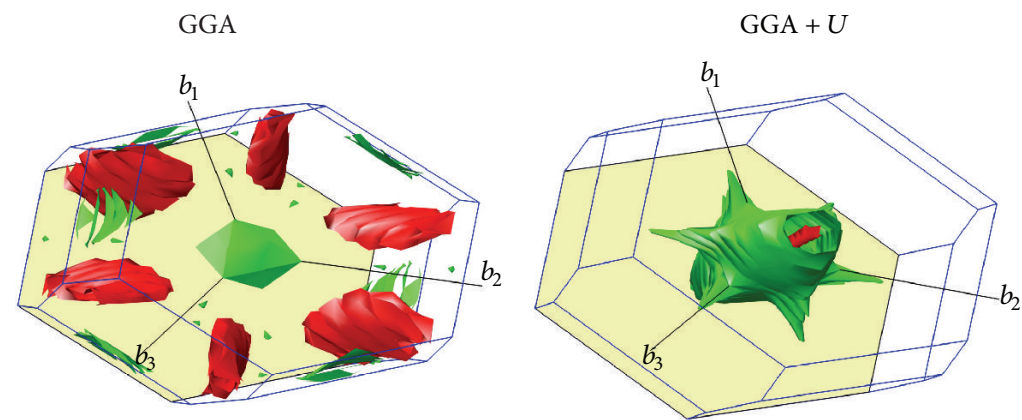

(a)
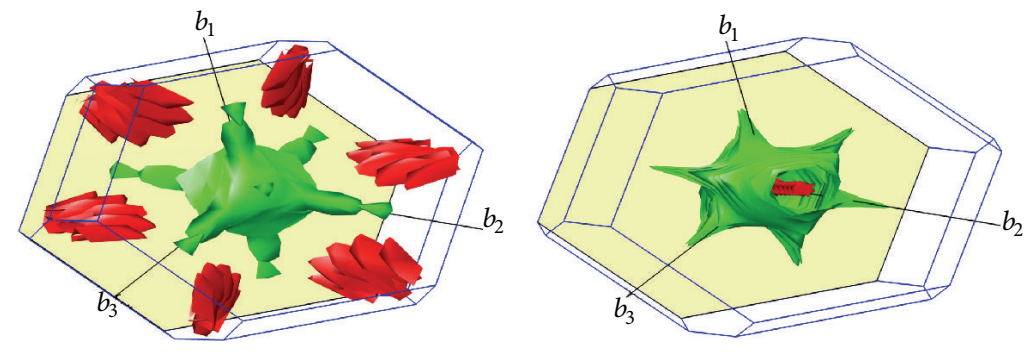

(b)
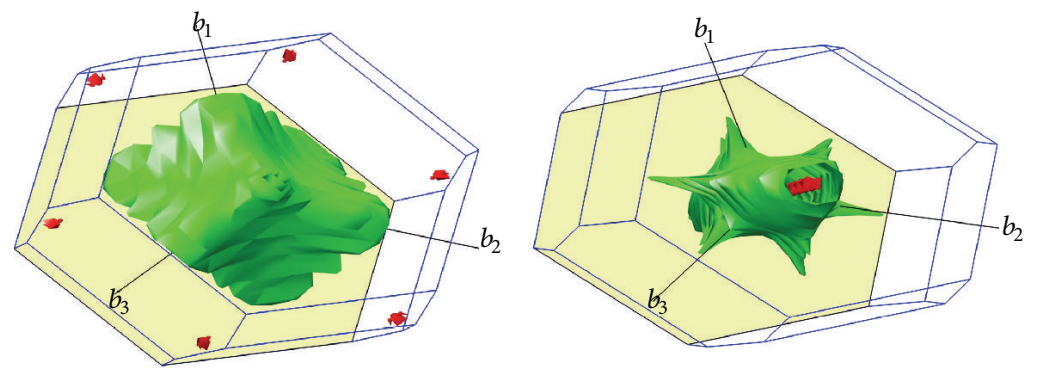

(c)

Figure 7: Fermi surface of CeS for GGA $+U$ with $U=6 \mathrm{eV}$.

are located in the approximate range $[-6-4] \mathrm{eV}$. The exact location of this group of bands moves closer to the Fermi level as one goes from CeS to $\mathrm{CeTe}$ by $0.5 \mathrm{eV}$ while the dispersion of the bands in the group remains almost the same. The states around the Fermi level for all three compounds are found to be formed mostly from cerium $4 \mathrm{f}$ states with a small contribution from the cerium $5 \mathrm{~d}$ orbitals as can be observed from the partial densities of states in Figures 5(a)-5(c). The very high density of states just above the Fermi level is due to dispersionless unoccupied f-levels of cerium atom.

The band structure and density of electronic states calculations were repeated in an GGA $+U$ framework to consider the effect of possible strong correlation effects related with the 4 f electrons of cerium. A $U_{\text {eff }}=6 \mathrm{eV}$ on Ce- 4 f orbitals is used. Its expected effect is to repel the occupied and unoccupied states down and up by $U_{\text {eff }} / 2$. The results are shown in Figures 6(a)-6(c) for $\mathrm{CeTe}, \mathrm{CeSe}$, and $\mathrm{CeS}$, respectively. Comparing GGA (Figure 5) and GGA + U (Figure 6), the most important effect of $+U$ correction is the decrease of density of states at the Fermi level originating from the $4 \mathrm{f}$ orbitals. GGA and GGA $+U$ band structures of Figures 5 and 6 can be compared with the similar calculations reported for CeS by [10]; while the band structure and the total density of states are very similar for our work and that of [10], the inclusion of $+U=$ $6 \mathrm{eV}$ correction was found to move the occupied $4 \mathrm{f}$ level $3 \mathrm{eV}$ below the Fermi level by Antonov et al. which is not found in the present work. The discrepancy is probably due to the difference in the implementation of Hubbard correction.

3.3. Fermi Surface. Fermi surfaces of CeS, CeSe, and CeTe are calculated at GGA and GGA $+U(U=6 \mathrm{eV})$ schemes and are displayed in Figures 7(a)-7(c), respectively. GGA + U Fermi surface of all three compounds are almost indistinguishable and are formed from two bands. The larger surface is formed from the bands originating from $\mathrm{Ce}-4$ f orbitals while the red thin cylindrical sector is due to the band formed from Ce$5 \mathrm{~d}$ electron states. The GGA Fermi surfaces (left column in Figure 7) of CeS and CeSe show some similarities.

3.4. Lattice Dynamics. The phonon dispersions of CeS, CeSe, and $\mathrm{CeTe}$ are obtained from linear response calculation and are displayed along with total and projected phonon density of states in Figures $8(\mathrm{a})-8(\mathrm{c})$, respectively. We also display the available experimental data for acoustical branch of 


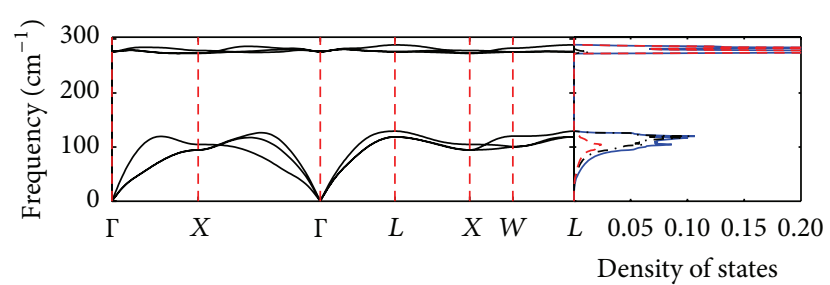

(a)

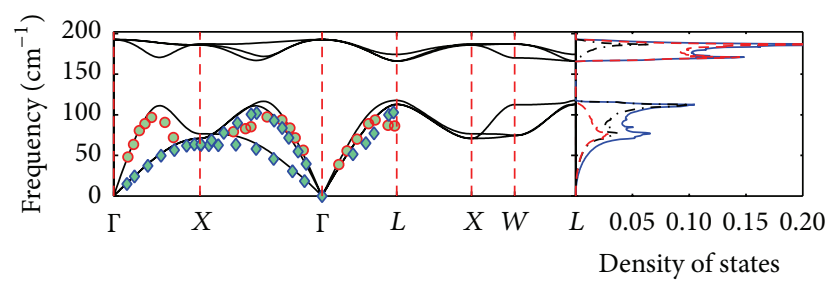

(b)

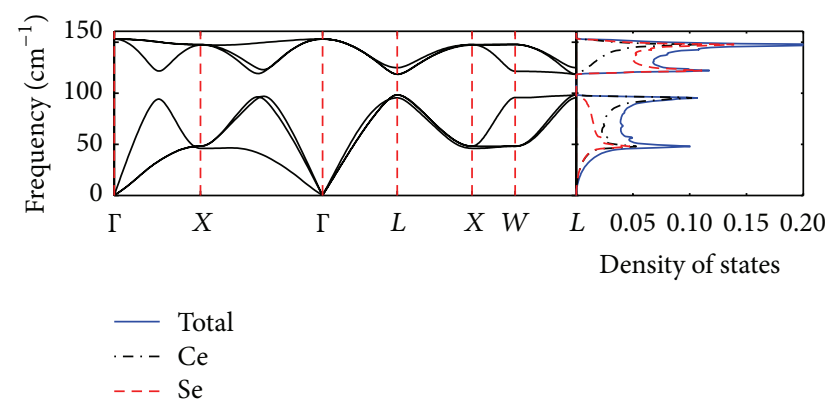

(c)

FIGURE 8: Phonon dispersions and the total and projected density of phonon states for (a) CeS, (b) CeSe, and (c) CeTe. The experimental acoustic mode frequencies of $\mathrm{CeSe}$ are shown with circle and diamonds and are from [2].

the phonons of CeSe [2] in Figure 8(b). The overall agreement between the experimental data and our calculated dispersion is good. While the flatness of the mode dispersions towards the zone boundaries is reproduced well in the computations, the crossing of the longitudinal and transverse acoustic modes near the L point is not reproduced for CeSe. The acoustical branch of the dispersions for all three compounds shows a similar structure; the maximum vibration frequency of the longitudinal acoustic modes reaches $\approx 100 \mathrm{~cm}^{-1}$ for wave vectors close to the zone boundary. Also, the contribution of chalcogenide atom to the displacement pattern of the acoustical modes decreases with increasing acoustical mode frequency while that of cerium contribution increases. The flatness of the dispersion around $\mathrm{X}$ point of the first Brillouine zone is mostly due to the motion of the chalcogenide atom and this flatness is inversely proportional to the mass of the chalcogenide.

On the other hand, the dispersion of the optical branch of the mode displays a richer structure compared to the acoustical ones. The displacement pattern of these modes mostly involves the motion of the chalcogenide atoms. The maximum frequency of the optical branch is around 300, 200, and $150 \mathrm{~cm}^{-1}$ for CeS, CeSe, and CeTe, respectively, and is inversely proportional to the square root of the mass of the chalcogenide atom. The optical modes of CeS are found to be almost dispersionless (Figure 8(a)). The width of optical mode dispersion is found to increase with the chalcogenide mass.

\section{Conclusion}

We have carried out an extensive first principles investigation of lattice, magnetic, electronic, and lattice dynamical properties of cerium monochalcogenides CeS, CeSe, and CeTe by employing density functional and density functional perturbation theory with GGA and Hubbard corrected GGA $+U$ exchange correlation functional.

The lattice constant and bulk modulus of all three compounds are found to be well described by GGA functional; adding $+U$ correction leads to an underbinding of the crystal which leads to an increase (decrease) in lattice constant (bulk modulus). The magnetic moment is found to be underestimated at the GGA level; GGA predicts ground state of $\mathrm{CeS}$ to be nonmagnetic. Inclusion of the correlation effect overestimates the magnetic moments. Both GGA and GGA + $U$ are unable to predict the experimentally observed magnetic moments of cerium monochalcogenides investigated in the present study.

We have found that the electronic bands close to the Fermi level have two distinct groups, at the Fermi level, bands are mostly cerium $4 \mathrm{f}$ character with a small Ce $5 \mathrm{~d}$ contribution while bands centered $5 \mathrm{eV}$ below the Fermi level are formed from a hybridization of cerium $5 \mathrm{~d}$ and chalcogenide $p$ levels. The density of electronic states at the Fermi level is found to be high at GGA level and decreases with the inclusion of strong correlation effects.

The lattice dynamical study indicates that the anomalous acoustical mode dispersion and the width of the dispersion of the optical mode branches strongly depends on the mass of the chalcogenide atom.

\section{Conflict of Interests}

The author declares that there is no conflict of interests regarding the publication of this paper.

\section{References}

[1] K. Hiraoka, R. Pittini, and P. Wachter, "Hall effect in Ce-monochalcogenides CeX (X = S, Se, Te)," Physica B: Condensed Matter, vol. 230-232, pp. 136-138, 1997.

[2] M. M. Steiner, H. Eschrig, and R. Monnier, "Longitudinalacoustic-phonon softening in YS, LaS, and CeSe," Physical Review B, vol. 45, no. 13, pp. 7183-7187, 1992.

[3] S. K. De and S. Chatterjee, "Electronic and optical properties of CeS under pressure," Physical Review B, vol. 40, no. 18, pp. 12304-12311, 1989.

[4] A. Svane, P. Strange, W. M. Temmerman, Z. Szotek, H. Winter, and L. Petit, "Pressure-induced valence transitions in rare earth chalcogenides and pnictides," Physica Status Solidi (b), vol. 223, no. 1, pp. 105-116, 2001. 
[5] D. L. Price, B. R. Cooper, S.-P. Lim, and I. Avgin, "Variation of the LDA $+U$ method appropriate to $f$-state localization: application to magneto-optical properties," Physical Review B, vol. 61 , no. 15 , p. $9867,2000$.

[6] D. Ravot, P. Burlet, J. Rossat-Mognod, and J. L. Tholence, "Magnetic properties of cerium monotelluride," Journal de Physique France, vol. 41, no. 10, pp. 1117-1120, 1980.

[7] P. Schobinger-Papamantellos, P. Fisher, A. Niggli, E. Kaldis, and V. Hildebrandt, "Magnetic ordering of rare earth monochalcogenides. I. Neutron diffraction investigation of CeS, NdS, NdSe, NdTe and TbSe," Journal of Physics C, vol. 7, p. 2023, 1973.

[8] R. Pittini, J. Schoenes, and P. Wachter, "MO Kerr study of the $4 \mathrm{f}^{1}$ state," Physica B: Condensed Matter, vol. 230-232, pp. 538-543, 1997.

[9] E. M. Collins, N. Kioussis, S. P. Lim, and B. R. Cooper, "Development of magnetism in strongly correlated cerium systems: non-Kondo mechanism for moment collapse," Physical Review B, vol. 62, Article ID 11533, 2000.

[10] V. N. Antonov, L. V. Bekenov, and A. N. Yaresko, "Electronic structure of strongly correlated systems," Advances in Condensed Matter Physics, vol. 2011, Article ID 298928, 107 pages, 2011.

[11] http://www.quantum-espresso.org/.

[12] J. P. Perdew, K. Burke, and M. Ernzerhof, "Generalized gradient approximation made simple," Physical Review Letters, vol. 77, no. 18, pp. 3865-3868, 1996.

[13] A. Svane, W. Temmerman, and Z. Szotek, "Theory of pressureinduced phase transitions in cerium chalcogenides," Physical Review B, vol. 59, no. 12, pp. 7888-7892, 1999.

[14] B. Coqblin, "Kondo lattice and magnetic properties in strongly correlated electron systems," Acta Physica Polonica A, vol. 115, no. 1, pp. 13-18, 2009.

[15] B. Amadon, F. Jollet, and M. Torrent, " $\gamma$ and $\beta$ cerium: LDA+U calculations of ground-state parameters," Physical Review B, vol. 77, no. 15, Article ID 155104, 2008.

[16] Local magnetic moments calculated using the spin polarized GGA minimum structure with GGA + U for $\mathrm{U}=6 \mathrm{eV}$.

[17] A. Bouhemadou, R. Khenata, M. Sahnoun, H. Baltache, and M. Kharoubi, "First-principles study of structural, elastic and high-pressure properties of cerium chalcogenides," Physica B, vol. 363, no. 1-4, pp. 255-261, 2005.

[18] J. M. Leger, "Chalcogenides and pnictides of cerium and uranium under high pressure," Physica B: Condensed Matter, vol. 190, no. 1, pp. 84-91, 1993.

[19] S. P. Lim, D. L. Price, and B. R. Cooper, "Giant magnetooptic rotations: the role of orbital polarization and explicit correlation effects," IEEE Transactions on Magnetics, vol. 27, no. 4, pp. 36483654, 1991.

[20] J. M. Léger, R. Epain, J. Loriers, D. Ravot, and J. Rossat-Mignod, "Anomalous behavior of CeTe under high pressures," Physical Review B, vol. 28, no. 12, pp. 7125-7129, 1983. 

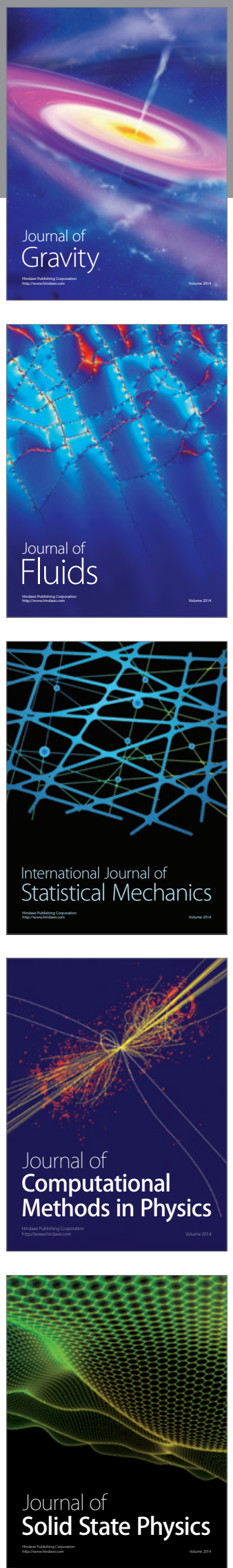

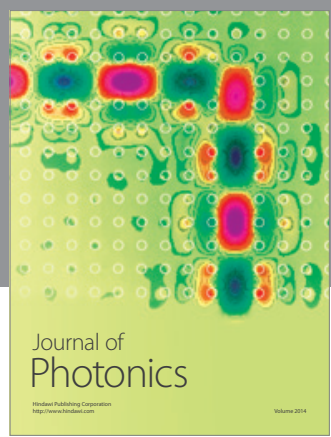

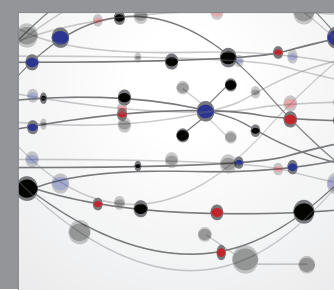

The Scientific World Journal

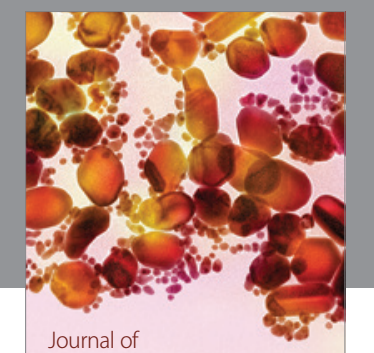

Soft Matter
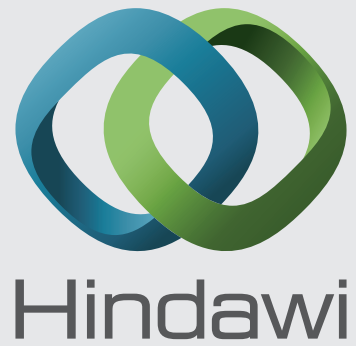

Submit your manuscripts at

http://www.hindawi.com
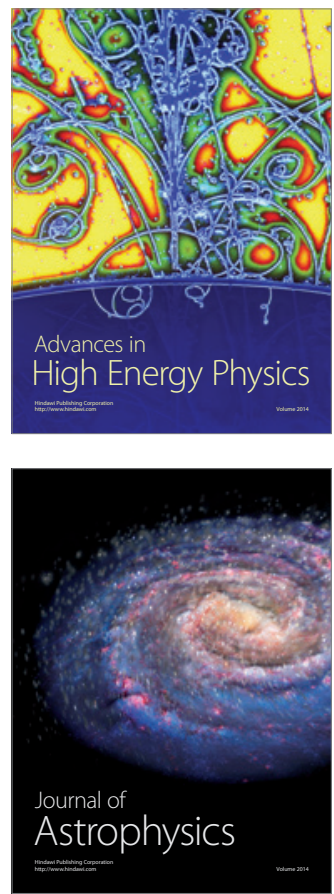
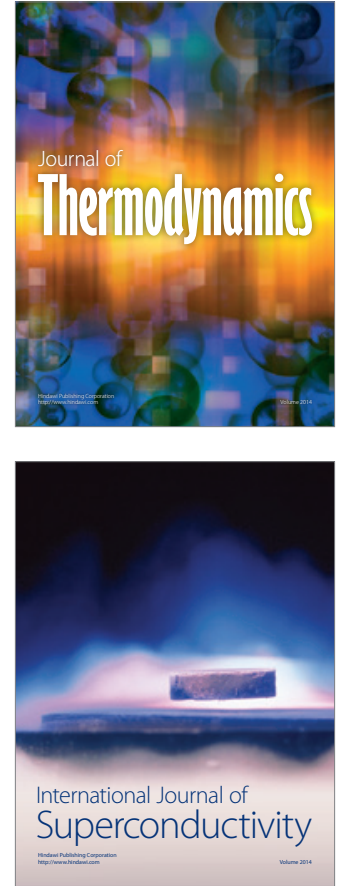
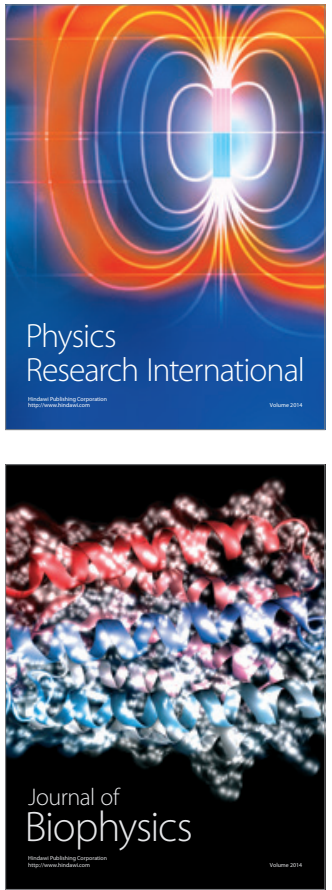
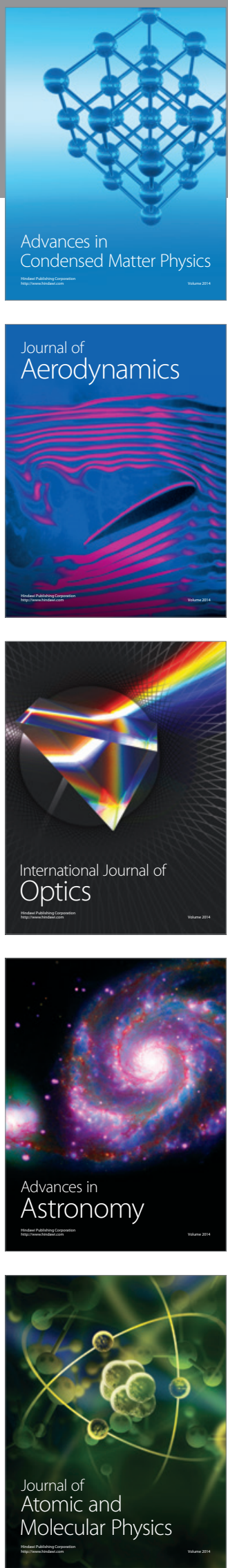